\title{
RESPUESTA FENOLÓGICA DE LA VEGETACIÓN ARBÓREA DE LAS LOMAS DEL SUR DEL PERÚ (MEJÍA - AREQUIPA) EN RELACIÓN CON EL EVENTO "EL NIÑO 1997-98"
}

\author{
PHENOLOGIC ANSWER OF THE ARBOREAL VEGETATION IN PERUVIAN SOUTHERN \\ LOMAS (MEJIA-AREQUIPA) IN RELATION TO "EL NIÑO 1997-98" EVENT
}

\author{
Carmelo Talavera, Percy Jiménez, Francisco Villasante, Aldo Ortega y Luis Villegas*
}

\section{RESUMEN}

Este trabajo presentó la evaluación del comportamiento fenológico de la vegetación arbórea con el fin de determinar su probable utilización como indicadores biológicos de la ocurrencia de eventos "El Niño". Los árboles evaluados fueron Caesalpinea spinosa, Duranta armata, Carica candicans y Citharexylum flexuosum. Los resultados indican una alta variación en las respuestas fenológicas. $C$. spinosa por ejemplo en 1995 y 1996 inició la floración antes que el brote; en 1997 inicio mucho antes, pero tuvo una corta duración, y en 1998, se ha presentado en forma tardía y breve, mientras que la cubierta foliar se ha mantenido en forma constante. En el caso de $D$. armata y $C$. flexuosum la floración se presenta posterior al brote, pero en ambos casos durante la ocurrencia del evento en 1998 no presentaron floración; y $C$. candicans presenta floración antes que el brote, pero con alta variabilidad en el tamaño de la floración.

Palabras claves: Fenologia, vegetación arbórea, El Niño, Lomas de Mejia, Arequipa.

\begin{abstract}
This work present the evaluation of phenologic behave of the arboreal vegetation in order to determine its possible use as biological indicator of "El Niño" events. The evaluated trees were Caesalpinia spinosa, Duranta armata, Carica candicans, and Citharexylum flexuosum. The results showed a high variation in phenologic answer. C. spinosa for example began flowering before shoot in 1995 and 1996; in 1997 began before, but had a short duration, and in 1998 it had presented in a late and brief period, while the foliage covering had been maintained constant. In the case of $D$. armata and $C$. flexuosum the flowering occurred after the shoot, but both cases during 1998 did not present flowering; and $C$. candicans presented flowering before the shoot, but with high variability in flowering size.
\end{abstract}

Key words: Phenology, arboreal vegetation, El Niño, Lomas of Mejia, Arequipa.

\section{INTRODUCCIÓN}

Los eventos "El Niño" como fenómeno climático oceanográfico atmosférico tienen como caracteristica principal el causar oscilaciones térmicas con gran incremento de temperatura en las aguas marinas en el Océano Pacífico. Éstas, al desplazarse hacia las costas de Sudamérica, por cambios en la dirección

\footnotetext{
* Instituto Regional de Ciencias Ambientales. Dpto. Acad. de Biología - UNSA
}

de los vientos alisios, causan importantes incrementos en las precipitaciones a lo largo de la faja costera de Ecuador, Perú y Chile. Los efectos no son los mismos en el continente, las abundantes precipitaciones en la costa norte del Perú, por ejemplo, pueden ser perjudiciales a los intereses humanos en el corto o mediano plazo, pero evidentemente serán beneficiosos en el largo plazo. En tanto, en la costa sur el incremento de la intensidad de nieblas y precipitaciones permiten el florecimiento del desierto en las formaciones 
de lomas permitiendo así tener un paisaje totalmente diferente al de los ecosistemas desérticos. Clarcke (1971), menciona por ejemplo los desastres causados por el evento "El Niño 1925" en las costas del Perú, así como los efectos positivos para la floración de la vegetación y el uso de ésta como forraje.

Los cambios ocurridos en los diferentes estadios de desarrollo de las plantas, como respuesta a las condiciones meteorológicas, precipitación y temperatura, podrían ser de importancia en la búsqueda de indicadores del evento. Por ello es que en el presente trabajo se hace el seguimiento de la fenología de la vegetación perenne en relación a los cambios climáticos.

\section{MATERIAL Y MÉTODOS}

Para el seguimiento de la fenología de la vegetación arbórea desde 1995 se instaló 4 transectos horizontales a los $500,600,700 \mathrm{y}$ $800 \mathrm{msnm}$, en estos se ha ubicado 10 plantas que corresponden a la vegetación arbórea, y las especies son: Caesalpinea spinosa, Duranta armata, Citharexilum flexuosum y Carica candicans.
En las evaluaciones mensuales desde abril de 1995 hasta agosto de 1998, se tomaron datos sobre estadíos de: reposo, brote, crecimiento y desarrollo o estado vegetativo, floración, fructificación y semilleo en pleno campo y anotados convenientemente fueron estudiados con rigurosidad.

Las condiciones meteorológicas, en los dos primeros años, fueron evaluadas con Data Logger que mide tres variables climáticas ( $p p$, dirección y velocidad de viento) y la temperatura con termómetro digital. Desde 1997 estas mediciones se realizan con una Estación Meteorológica Campbell (Jiménez et al., en este volumen).

\section{RESULTADOS}

La Tab. 1 muestra los resultados de las evaluaciones meteorológicas (temperatura y precipitación) correspondientes a los años de estudio; se destaca una regularidad en las condiciones de temperatura de la zona y una alta variabilidad en las precipitaciones con una tendencia a incrementarse constantemente, conforme se avanza hacia la ocurrencia del evento "El Niño 1997-98".

Tabla 1. Valores de temperatura y precipitación promedio mensual para el periodo 19951998 en las lomas de Mejia (Mollendo - Arequipa).

\begin{tabular}{|c|c|c|c|c|c|c|c|c|}
\hline \multirow[t]{3}{*}{ Mes } & \multicolumn{8}{|c|}{ Año } \\
\hline & \multicolumn{2}{|c|}{1995} & \multicolumn{2}{|c|}{1996} & \multicolumn{2}{|c|}{1997} & \multicolumn{2}{|c|}{1998} \\
\hline & $t\left({ }^{\circ} \mathrm{C}\right)$ & $\mathrm{pp}(\mathrm{mm})$ & $t\left({ }^{\circ} \mathrm{C}\right)$ & $\mathrm{pp}(\mathrm{mm})$ & $t\left({ }^{\circ} \mathrm{C}\right)$ & $\mathrm{pp}(\mathrm{mm})$ & $t\left({ }^{\circ} \mathrm{C}\right)$ & $\mathrm{pp}(\mathrm{mm})$ \\
\hline Enero & & & 21,9 & 0 & 22,5 & 15,2 & 18,7 & 173,6 \\
\hline Febrero & & & 22,1 & s.d & 19,3 & 3,2 & 19,1 & 75,4 \\
\hline Marzo & & & 21,5 & s.d & 19,5 & 6,0 & 18,6 & 23,0 \\
\hline Abril & & & 18,2 & s.d & 16,7 & 0,8 & 17,4 & 5,0 \\
\hline Mayo & 18,3 & & 17,5 & s.d & 15,6 & 6,8 & 14,9 & 2,0 \\
\hline Junio & 17,2 & & 14,5 & s.d & 13,6 & 3,8 & 13,5 & 11,4 \\
\hline Julio & 15,3 & & 14,7 & s.d & 13,3 & 23,4 & 12,3 & 15,4 \\
\hline Agosto & 13,6 & & 13,7 & 37,2 & 14,4 & 61,0 & & \\
\hline Setiembre & 14,5 & 28,0 & s.d & 4,4 & 14,4 & 189,4 & & \\
\hline Octubre & 16,2 & 15,0 & 16,2 & 2,4 & 14,1 & 66,6 & & \\
\hline Noviembre & 18,7 & 1,6 & 14,9 & 5,4 & 14,7 & 41,8 & & \\
\hline Diciembre & 19,4 & 1,6 & 21,1 & 0,4 & 16,7 & 84,6 & & \\
\hline prom. t $\left({ }^{\circ} \mathrm{C}\right)$ & 16,6 & & 17,8 & & 16,2 & & 16,3 & \\
\hline pp total (mm) & & 46,2 & & 49,8 & & 502,6 & & 305,8 \\
\hline
\end{tabular}


Tabla 2. Estado fenológico de la vegetación arbórea en las lomas de Mejía (Mollendo Arequipa) en 1995.

\begin{tabular}{|c|c|c|c|c|c|c|c|c|c|c|}
\hline \multirow{2}{*}{ Especie } & \multirow{2}{*}{$\begin{array}{l}\text { Estado } \\
\text { fenológico }\end{array}$} & \multicolumn{9}{|c|}{ Período de evaluación } \\
\hline & & $\mathrm{Abr}$ & May & Jun & Jul & Ago & Set & Oct & Nov & Dic \\
\hline \multirow[t]{5}{*}{ C. spinosa } & Reposo & + & + & & & & & + & + & + \\
\hline & Brote & & & & & & + & + & + & \\
\hline & Floración & & & & & + & + & & & \\
\hline & Fructificación & & & & & & + & + & & \\
\hline & Semilleo & & & & & & & + & + & + \\
\hline \multirow[t]{5}{*}{ D. armata } & Reposo & + & + & + & & & & + & + & + \\
\hline & Brote & & & & & & + & + & + & \\
\hline & Floración & & & & & + & + & & & \\
\hline & Fructificación & & & & & & + & + & + & \\
\hline & Semilleo & & & & & & & + & + & \\
\hline \multirow[t]{5}{*}{ C. flexuosum } & Reposo & + & + & + & + & & & + & + & + \\
\hline & Brote & & & & & & & & & \\
\hline & Floración & & & & & & & + & & \\
\hline & Fructificación & & & & & & & & + & \\
\hline & Semilleo & & & & & & & & & \\
\hline \multirow[t]{5}{*}{ C. candicans } & Reposo & & + & + & + & & & & & \\
\hline & Brote & & & & & & + & + & & \\
\hline & Floración & & & & & + & + & & & \\
\hline & Fructificación & & & & & & + & + & & \\
\hline & Semilleo & & & & & & & & & \\
\hline
\end{tabular}

En la Tab. 2 se registra que en 1995 todas las especies normalmente empezaron la floración antes que el brote. Sin embargo, la duración de las fases fue diferente entre las especies, así como el momento en que inician la respuesta fenológica a las condiciones climáticas. Tanto C. spinosa como D. armata presentaron una floración bastante distanciada en el tiempo con respecto al brote, mientras C. flexuosum no presentó esta fase y la floración fue de corta duración.

En 1996, C. spinosa presentó floración en forma tardía con respecto a 1995 como se observa en la Tab. 3, asimismo la diferencia entre el tiempo de inicio del brote con el de la floración es más corta. D. armata, por otro lado, mostró una diferencia más larga entre estas dos fases. $C$. flexuosum al igual que $C$. spinosa también mostró una respuesta tardía, y contradictoriamente $C$. candicans inicia primero su brote antes que la floración y ésta fue de corta duración.
En 1997, todas las especies evaluadas, excepto $C$. flexuosum, iniciaron sus fases de desarrollo en forma temprana, adelantando tanto la floración como el brote. C. spinosa presentó una fase de floración bastante larga pero a inicios de agosto la perdió, sin haber iniciado la fructificación y no volvió a formar flores por el resto del año. Sin embargo, el brote y formación de la cubierta foliar permanecieron constantes. En el caso de $D$. armata y $C$. candicans, ambas perdieron la floración y el brote en agosto y permanecieron en ese estado por el resto del período; y $C$. flexuosum presentó una floración y brote tardíos y de corta duración (Tab. 4).

En 1998, todas las especies permanecieron en reposo hasta julio en que $C$. candicans inició la floración y a partir de setiembre las demâs especies mostraron indicios de actividad (Tab. 5). Asî es notorio que $C$. spinosa mantuvo una cubierta foliar constante, D. armata inició el brote antes que la floración 
Tabla 3. Estado fenológico de la vegetación arbórea en las lomas de Mejía (Mollendo * Arequipa) en 1996.

\begin{tabular}{|c|c|c|c|c|c|c|c|c|c|c|c|c|c|}
\hline \multirow[t]{2}{*}{ Especie } & \multirow{2}{*}{$\begin{array}{l}\text { Estado } \\
\text { fenológico }\end{array}$} & \multicolumn{12}{|c|}{ Período de evaluación } \\
\hline & & Ene & Feb & Mar & Abr & May & Jun & Jul & Ago & Set & Oct & Nav & Dic \\
\hline \multirow[t]{5}{*}{ C. spinosa } & Reposo & + & + & + & + & + & + & + & & & & & + \\
\hline & Brote & & & & & & & + & + & + & & & \\
\hline & Floración & & & & & & & + & + & + & & & \\
\hline & Fructificación & & & & & & & & & + & + & + & + \\
\hline & Semilleo & & & & & & & & & & + & + & + \\
\hline \multirow[t]{5}{*}{ D. armata } & Reposo & + & + & + & + & & & & + & + & + & + & + \\
\hline & Brote & & & & & & & + & + & + & + & & \\
\hline & Floración & & & & & & + & + & + & + & & & \\
\hline & Fructificacion & & & & & & & & + & + & + & & \\
\hline & Semilleo & & & & & & & & & + & + & + & \\
\hline \multirow[t]{5}{*}{ C. flexuosum } & Reposo & + & + & + & + & + & + & + & + & + & & + & + \\
\hline & Brote & & & & & & & & & + & + & & \\
\hline & Floración & & & & & & & & + & + & & & \\
\hline & Fructificación & & & & & & & & & + & + & & \\
\hline & Semilleo & & & & & & & & & & & & \\
\hline \multirow[t]{4}{*}{ C. candicans } & Reposo & + & + & + & + & + & & & & & + & + & + \\
\hline & Brote & & & & & + & + & + & + & + & + & + & \\
\hline & Floración & & & & & & + & + & & & & & \\
\hline & $\begin{array}{l}\text { Fructificación } \\
\text { Semilleo }\end{array}$ & & & & & & & & + & + & + & + & \\
\hline
\end{tabular}

y C. flexuosum recién empezó a mostrar indicios de brote.

\section{DISCUSIÓN}

Desde 1995, inicio de la presente investigación, las condiciones climatológicas en las lomas de Mejía han ido variando, de condición seca hacia otra más húmeda. Los años 1995 y 1996 fueron secos en términos de precipitación, lo que se considera como años normales, puesto que las precipitaciones no son muy diferentes a las que registró la Estación de la Marina de Guerra del Perú en Punta Islay, que es de 10,8 mm (Flores, 1997).

Tabla 4. Estado fenológico de la vegetación arbórea en las lomas de Mejía (Mollendo Arequipa) en 1997.

\begin{tabular}{|c|c|c|c|c|c|c|c|c|c|c|c|c|c|}
\hline \multirow[t]{2}{*}{ Especie } & \multirow{2}{*}{$\begin{array}{l}\text { Estado } \\
\text { fenológico }\end{array}$} & \multicolumn{12}{|c|}{ Período de evaluación } \\
\hline & & Ene & $\mathrm{Feb}$ & Mar & $\mathbf{A b r}$ & May & Jun & Jul & Ago & Set & Oct & Nov & Dic \\
\hline \multirow[t]{5}{*}{ C. spinosa } & Reposo & + & + & + & + & & & & & & & & \\
\hline & Brote & & & & & + & + & + & + & + & + & + & + \\
\hline & Floración & & & + & + & + & + & + & + & & & & \\
\hline & Fructificación & & & & & & & & & & & & \\
\hline & Semilleo & & & & & & & & & & & & \\
\hline \multirow[t]{5}{*}{ D. armata } & Reposo & + & + & + & + & & & & + & + & + & + & + \\
\hline & Brote & & & & & & + & + & + & & & & \\
\hline & Floración & & & & & + & + & + & & & & & \\
\hline & Fructificación & & & & & & & & & & & & \\
\hline & Semilleo & & & & & & & & & & & & \\
\hline \multirow[t]{5}{*}{ C. flexuosum } & Reposo & + & + & + & + & + & + & + & + & & + & + & + \\
\hline & Brote & & & & & & & & & + & + & & \\
\hline & Floración & & & & & & & & + & + & & & \\
\hline & Fructificación & & & & & & & & & & & & \\
\hline & Semilleo & & & & & & & & & & & & \\
\hline \multirow[t]{5}{*}{ C. candicans } & Reposo & + & + & + & & & + & + & + & + & + & + & + \\
\hline & Brote & & & & & + & + & + & + & & & & \\
\hline & Floración & & & & + & + & & & & & & & \\
\hline & Fructificación & & & & & & & & & & & & \\
\hline & Semilleo & & & & & & & & & & & & \\
\hline
\end{tabular}


Tabla 5. Estado fenológico de la vegetación arbórea en las lomas de Mejía (Mollendo Arequipa) en 1998.

\begin{tabular}{|c|c|c|c|c|c|c|c|c|c|c|}
\hline \multirow[t]{2}{*}{ Especie } & \multirow{2}{*}{$\begin{array}{l}\text { Estado } \\
\text { fenológico }\end{array}$} & \multicolumn{9}{|c|}{ Período de evaluación } \\
\hline & & Ene & Feb & Mar & Abr & May & Jun & Jul & Ago & Set \\
\hline \multirow[t]{2}{*}{ C. spinosa } & Reposo & + & + & + & + & + & + & + & & \\
\hline & $\begin{array}{l}\text { Brote } \\
\text { Floración } \\
\text { Fructificación } \\
\text { Semilleo }\end{array}$ & + & + & + & + & + & + & + & + & $\begin{array}{l}+ \\
+\end{array}$ \\
\hline D. armata & $\begin{array}{l}\text { Reposo } \\
\text { Brote } \\
\text { Floración } \\
\text { Fructificación } \\
\text { Semilleo }\end{array}$ & & & + & + & + & + & + & + & $\begin{array}{l}+ \\
+\end{array}$ \\
\hline C. flexuosum & $\begin{array}{l}\text { Reposo } \\
\text { Brote } \\
\text { Floración } \\
\text { Fructificación } \\
\text { Semilleo }\end{array}$ & & & + & + & + & + & + & + & $\begin{array}{l}+ \\
+\end{array}$ \\
\hline \multirow[t]{4}{*}{ C. candicans } & Reposo & & + & + & + & + & & & & \\
\hline & Brote & & & & & & & + & + & + \\
\hline & Floración & & & & & & + & + & & \\
\hline & $\begin{array}{l}\text { Fructificación } \\
\text { Semilleo }\end{array}$ & & & & & & & + & + & + \\
\hline
\end{tabular}

En los siguientes años 1997 y 1998, las condiciones climáticas variaron completamente, el ambiente se hizo más húmedo, las precipitaciones en las lomas alcanzaron los $500 \mathrm{~mm}$ en 1997 y más de 300 mm entre enero y marzo de 1998. Estas condiciones hacen propicia la floración de la vegetación herbácea y una respuesta vegetativa de la arbórea y arbustiva.

Las respuestas fenológicas de las especies evaluadas no sólo son diferentes entre ellas, sino también con respecto a un período. Es notable observar que en el caso de Caesalpinea spinosa, muestra un desarrollo de todas sus fases en 1995 y 1996, esto probablemente provocado por un ligero incremento en las precipitaciones. Durante 1997 adelanta su floración sin haber un mayor incremento significativo de la humedad, alcanzando una abundante floración y brote foliar. Sin embargo, desde agosto de 1997 y parte de 1998, período en que ocurre el evento climático "El Niño", esta especie sólo mostró brote o mantuvo su cobertura foliar, mientras que los signos de inicio de la floración, para la presente temporada, recién se hacen evidentes a partir de setiembre.

Con relación a $D$. armata y $C$. flexuosum, los momentos de respuesta a los factores ambientales presentan alta variacion en temporalidad, así vemos que para 1995 tanto el brote como la floración coincidieron con la temporada húmeda; para 1996 tanto la floración como el brote foliar se hicieron más evidentes y con mayor intensidad y duración que el año anterior, mientras que para 1997-98 no respondieron favorablemente al incremento de humedad.

Carica candicans, especie frutícola que crece en lomas, también, muestra un comportamiento diferencial en el tiempo, así para 1995 y 1996 el brote y la floración ocurrieron antes del inicio del período húmedo; en 1996 estas dos fases fueron más largas que el año anterior y característicamente más abundantes en flores y hojas, mientras que en 1997 no presentó floración y en 1998 tanto el brote como la floración se presentaron en 
forma más tardía con respecto a 1995 y 1996.

En todos los casos la floración se presentó antes que el brote de las hojas, la caída de las hojas ocurrió antes de la maduración del fruto y la caída de los frutos ocurrió mucho después de que el árbol entre en agoste. Adicionalmente, esta forma de respuesta que presentaron las especies evaluadas evidentemente estuvo en relación con las condiciones ambientales del momento, por lo que hay una posibilidad de ser utilizadas como indicadores biológicos. Sin embargo, se requiere de un mayor seguimiento y realizar estudios comparativos con otras áreas donde existen las mismas especies.
Agradecimientos: A la Unión Europea (Convenio UNSA - PADOVA), la Organización de los Estados Americanos (OEA), el Consejo Nacional de Ciencia y Tecnología (CONCYTEC) y a la Red RIBEN, por haber patrocinado y financiado esta investigación.

\section{LITERATURA CITADA}

Clarcke, G. 1971. Ecología general. Edit. Omega. Barcelona. XIV+534 pp.

Flores, R. 1997. Análisis de la degradación y estado actual de las lomas de Mejía (Islay/Arequipa. Julio 1996 a Febrero 1997). Arequipa (Perú). Tesis para optar el Título de Biólogo. Univ. Nac. San Agustín, Arequipa. Perú. 73 pp. 